\title{
Optical Characteristics Analysis of Resonant Tunneling Diode Photodiode based Oscillators
}

\author{
Weikang Zhang ${ }^{1}$, Scott Watson ${ }^{1}$, Jue Wang ${ }^{1}$, José Figueiredo ${ }^{2}$, Edward Wasige ${ }^{1}$, Anthony E. Kelly ${ }^{1}$ \\ ${ }^{I}$ School of Engineering, University of Glasgow, Glasgow, United Kingdom \\ ${ }^{2}$ Departamento de Física, CEOT, Universidade do Algarve, Portugal \\ email:w.zhang.1@research.gla.ac.uk
}

\begin{abstract}
This paper represents the experimental results of optical modulation characteristics of a resonant tunneling diodephotodiode (RTD-PD) device and novel microwave oscillators employing such RTD-PD. Different from a pure electronic RTD oscillator, the RTD-PDs in this work contain photoconductive layers which make it able to operate in optical conditions. A beam of intensity modulated light with a wavelength of $1310 \mathrm{~nm}$ was sent to the free running oscillator via an optical fiber whilst the RTD device was biased in the oscillation condition, and the corresponding electrical data can be extracted from the output port of the oscillator. A square wave signal at data rates up to 10 Gbit/s, and a pseudo random binary sequence (PRBS) signal with a data rate of $100 \mathrm{Mbit} / \mathrm{s}$ were preliminarily implemented for modulation.
\end{abstract}

Keywords-Resonant tunneling diode-photodiode, Terahertz, optical communication.

\section{INTRODUCTION}

The demand for bandwidth in both wired and wireless communications is pressing under the pressure of evergrowing high bandwidth services and it is expected that wireless data rates in the order of tens of Gbit/s will be desired in the near future [1]. In consideration of spectrum efficiency, it is necessary to utilize higher frequency bands such as terahertz (THz), which lie between millimeter-wave and infrared light, above $60 \mathrm{GHz}$ and up to $1 \mathrm{THz}$. It is still rare in the scope of wireless communication links because of the lack of suitable devices capable of generating and detecting $\mathrm{THz}$ waves. Resonant tunneling diodes (RTDs) have been explored for decades to achieve $\mathrm{THz}$ oscillation at room temperature. They have been reported as the fastest purely electronic devices with oscillation frequencies above $1.9 \mathrm{THz}$ at room temperature [2].

RTDs are devices implemented on monolithic microwave integrated circuit (MMIC) technology with a compact structure which is less than a millimeter square. RTDs provide an intrinsic gain benefited from the negative differential conductance (NDC) region and require much lower power consumption compared with traditional transistor technologies. Thanks to the similar material system, it makes them possible to be integrated with optoelectronic components seamlessly such as photodetectors [3]. These advantages make the implementation using RTDs simple and efficient without complex transceiver building components [3]. Therefore, the opto-electronic RTD is suitable to be integrated into high speed fiber-optic networks in future communication systems. $300-\mathrm{GHz}$ wireless transmission at data rate of $2.5 \mathrm{Gbit} / \mathrm{s}$ at 1 meter transmission distance has been reported using electronic RTDs [4, 5]. It is still novel to implement high speed RTD based oscillators in optical communication as a photodetector even though intensive works have been done in the area of optoelectronics based on RTD devices such as opto-modulator $[6,7]$, RTD-photosensor [8] and optical injection locking experiments [9-11].

This paper represents experimental results on an individual RTD-PD device and RTD-PD oscillators. They are utilized to photo detect an optical signal modulated by a sequential square wave signal with a data rate up to $10 \mathrm{Gbit} / \mathrm{s}$ and a PRBS signal with a data rate of $100 \mathrm{Mbit} / \mathrm{s}$. A similar experiment has been conducted previously using a $2.8 \mathrm{GHz}$ RTD device with $75 \mathrm{MHz}$ modulation data rate [12]. Here, an RTD-PD oscillator with an oscillation frequency of $5.47 \mathrm{GHz}$ is used for preliminary tests, before an oscillator at $35.1 \mathrm{GHz}$ was implemented.

\section{SYSTEM DESCRIPTION}

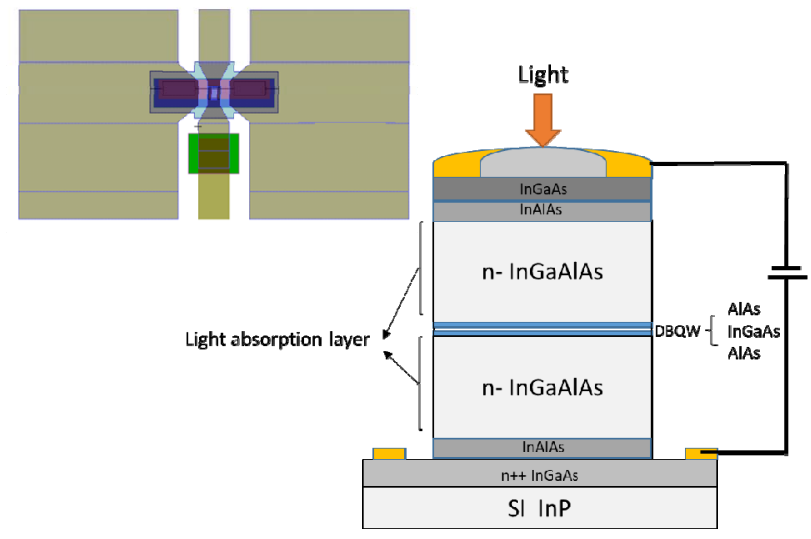

(a) 


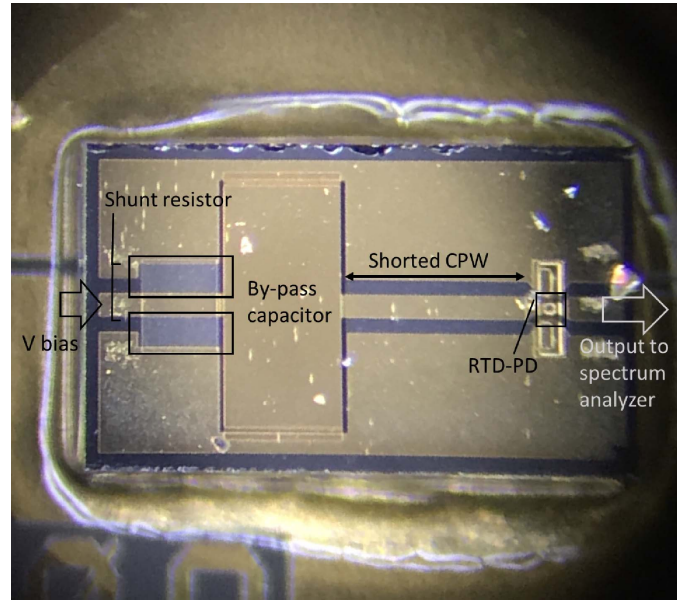

(b)

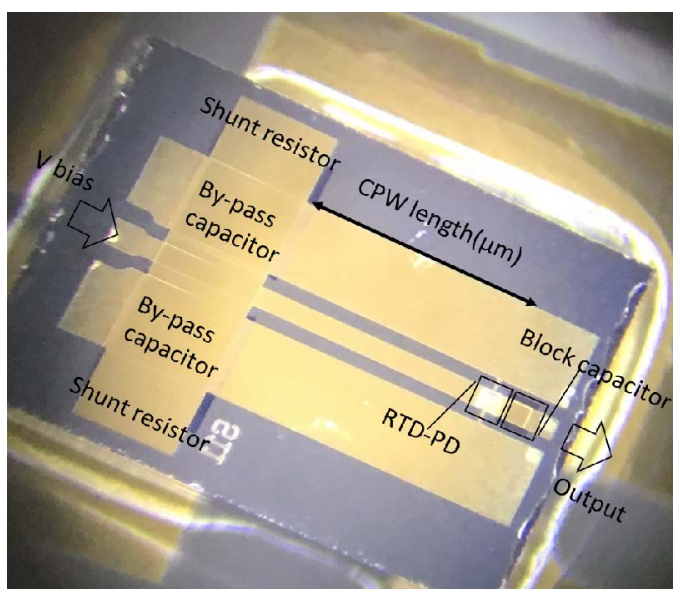

(c)

Fig. 1. (a) individual RTD-PD device surrounded by electrode pads(left) and RTD-PD epi-layer structure (right), (b) photo of the 5.47 GHz RTD-PD oscillator, (c) photo of the $35.1 \mathrm{GHz}$ RTD-PD oscillator.

Fig. 1(a) shows the layout of an individual RTD-PD device and the epi-layer structure of the RTD-PD. The device under test has a device size of $24 \times 24 \mu \mathrm{m}^{2}$ and window size $24 \times 11$ $\mu \mathrm{m}^{2}$. The light absorption layers are located at both sides of the double barrier quantum well (DBQW). Fig. 1(b) and (c) show the photos of the oscillators with different oscillation frequencies. The oscillators under test employ one RTD-PD connected to a shorted $50 \Omega$ coplanar waveguide (CPW) which is shorted by a bypassing capacitor. A stabilizing shunt resistor, made of thin film $\mathrm{NiCr}$, is to suppress the low frequency bias oscillations. The bypassing capacitor, which is realized as a metal-insulator-metal (MIM) capacitor, is designed to shortcircuit the RF signal to ground to avoid dissipation of RF power over the stabilizing resistor [13]. There is a block capacitor in the output side used to prevent any DC leakage flowing into the measurement equipment during on-wafer measurements. The targeted oscillation frequency is dependent on the inductance of the CPW line and the RTD selfcapacitance. It can be tuned by the DC bias across the NDC as consequence of changing the RTD impedance. It makes such circuit as a voltage controlled oscillator.
When an intensity modulated light pulse illuminates the active area of the RTD-PD, which is biased close to the peak current point of the NDC, photo-charges are produced and the internal resistance will be reduced. It leads to an increase of current and a shift of the I-V curve. Due to this mechanism, it switches the quiescent operation point into the NDC region giving rise to a current change in the external circuit [14].

\section{EXPERIMENTAL SET-UP AND RESULTS}

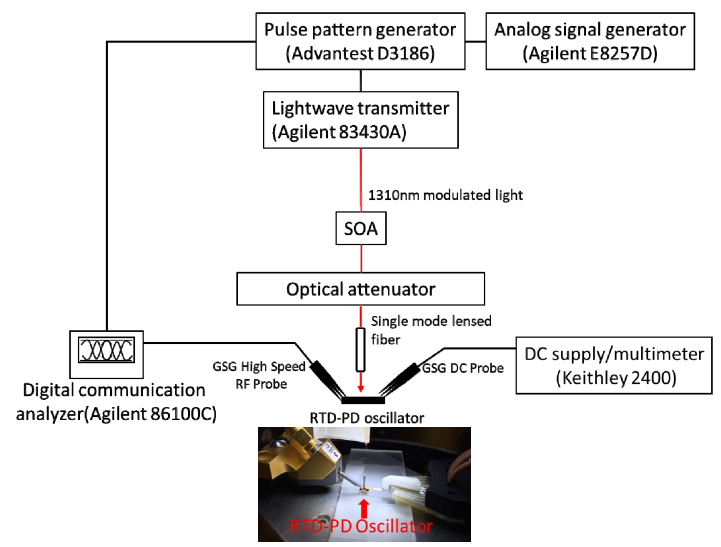

Fig. 2. experimental set-up for optical modulation

On-wafer measurements illustrated in Fig. 2 were carried out to characterize the RTD-PD device and oscillator. A laser beam, with a wavelength of $1310 \mathrm{~nm}$, was intensity modulated by a signal generated from a pulse pattern generator and its data rate could be chosen by the signal generator. The pattern used as the testing signal was a square wave containing alternant ' 0 ' and ' 1 ' levels. PRBS tests have also been implemented. The modulated optical signal was then amplified by a semiconductor optical amplifier (SOA). An RF groundsignal-ground (GSG) probe was used to probe the output port of the oscillator and the output signal was extracted to the digital communication analyser or spectrum analyzer. A single mode lensed fiber was aligned with the RTD-PD, at an almost vertical angle to the optical access windows. The optimized alignment could be confirmed by acquiring the largest photo current value on the multimeter.

1) Individual RTD-PD device

This part demonstrates the measurement based on the individual RTD-PD device which has been shown in Fig. 1(a). Firstly, a typical current-voltage (I-V) characteristic under dark and light conditions (continuous wave (CW) light at wavelength of $1310 \mathrm{~nm}$ and $1550 \mathrm{~nm}$, and modulated $1310 \mathrm{~nm}$ optical carrier) is measured shown in Fig. 3. Since a large amount of the injected photons is absorbed in the emitter side of the device. The I-V curve was shifted to higher voltage (right side) and also the current increases. Because the material employed in the optical absorption layer has better absorption efficiency at $1310 \mathrm{~nm}$ than $1550 \mathrm{~nm}$, the photocurrent is higher for $1310 \mathrm{~nm} \mathrm{CW}$ light. 


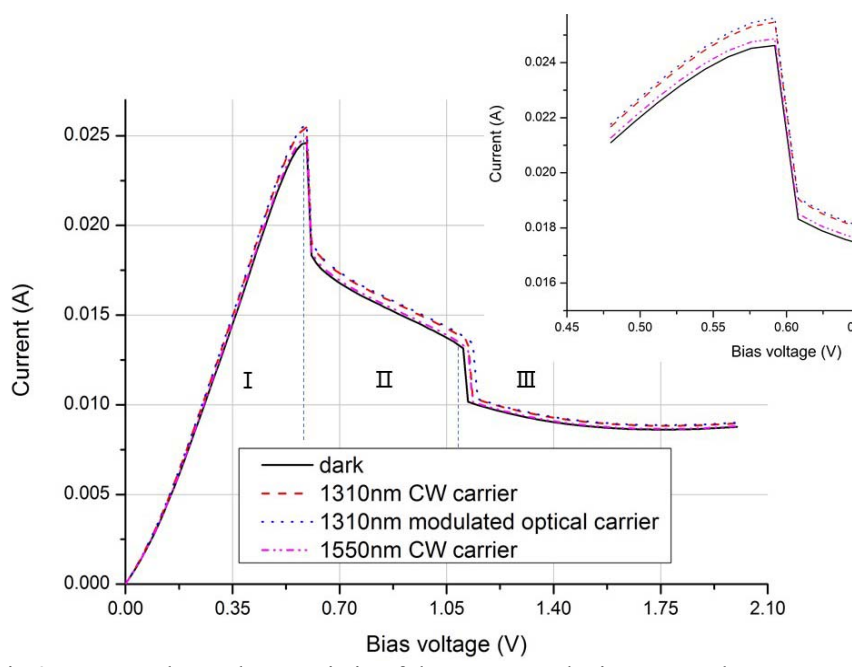

Fig.3. Measured I-V characteristic of the RTD-PD device. Inset: the I-V curve shifting to the right in the presence of light.

With the modulated optical carrier at a wavelength of $1310 \mathrm{~nm}$, data transmission was implemented in three bias regions. The first bias region is from $0 \mathrm{~V}$ to $0.591 \mathrm{~V}$, which is in the first positive differential resistance (PDR) region before the peak current. The second region is from $0.591 \mathrm{~V}$ to $1.08 \mathrm{~V}$, corresponding to the NDR region, and the third region is beyond the NDR region. The optical carrier was modulated with a PRBS pattern at $50 \mathrm{Mbit} / \mathrm{s}$ and $100 \mathrm{Mbit} / \mathrm{s}$ data rate. Table 1,2 and 3 below show the eye patterns, output amplitudes and corresponding oscillation frequencies when biased in region 2 .

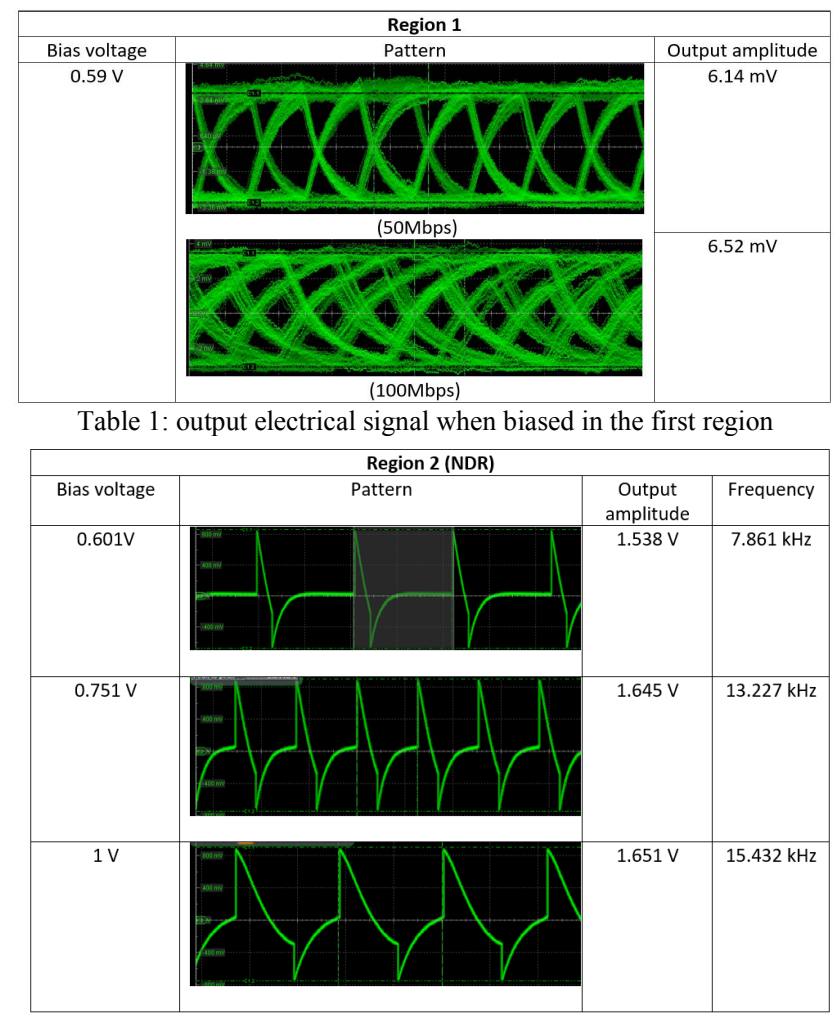

Table 2: self-oscillation when biased in the second (NDR) region

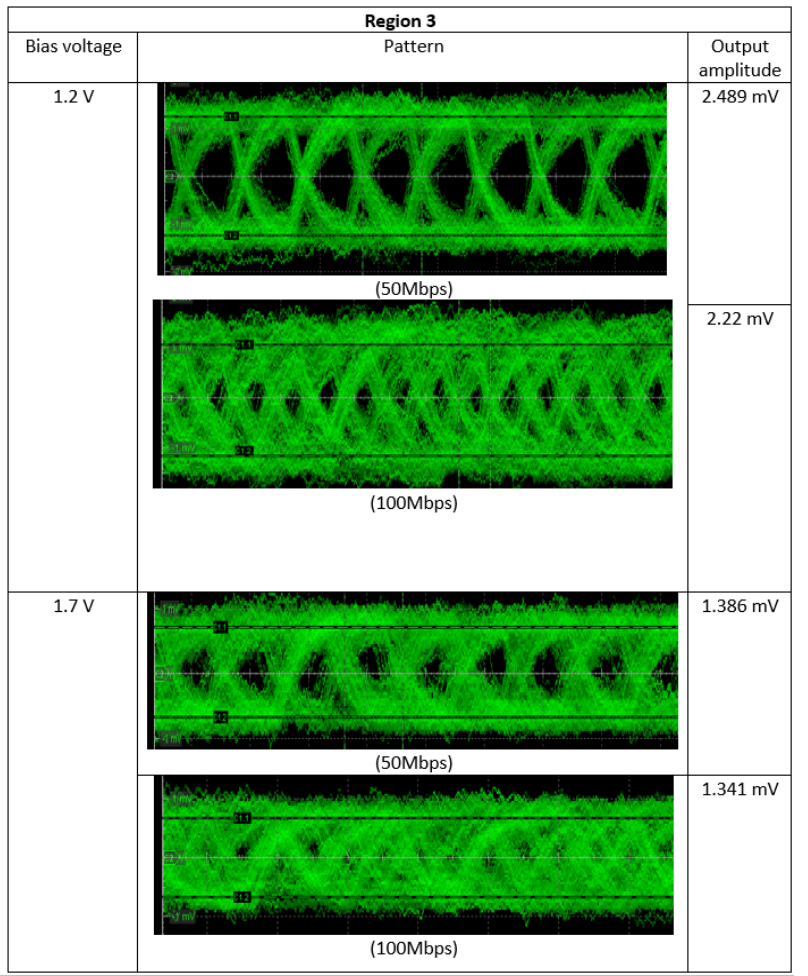

Table 3: output electrical signal when biased in the third region

It was observed that the output electrical signal could present eye diagrams in the whole PDR region, and the best eye diagrams were observed at the bias just before the NDR region because of higher output power and better optical absorption. In the second bias region, the eye disappeared and optical injection played a role as an additional bias voltage, which tuned the oscillation frequency. The modulated light would not give significant impact on the oscillation frequency or output amplitude. Besides, the output amplitude was high, with amplitudes up to $1.65 \mathrm{~V}$. The shape and frequency of the oscillation pulse were remarkably dependent on the external connection elements such as bias-T and cables. When biased in the third region, the eye appeared again but with lower output amplitude compared with the first region.

To further explore the correlation between the selfoscillation and the bias voltage, Fig.4 below represents the variation of frequency and output signal voltage according to the bias in the second region.

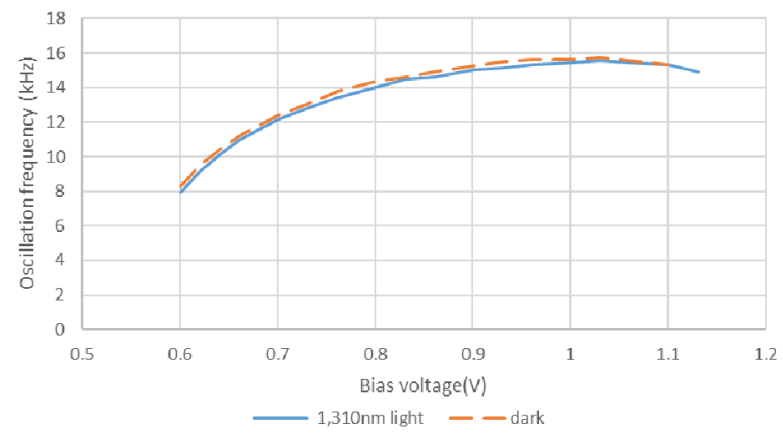

Fig. 4. (a): self-oscillation frequency when biased in the NDR region in dark and light conditions. 


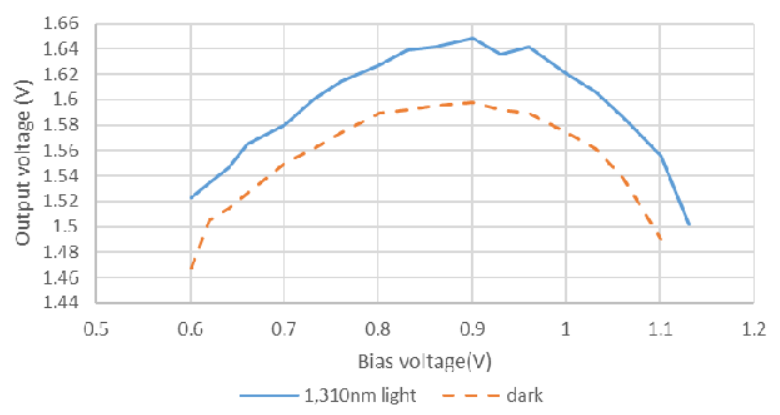

Fig. 4. (b): amplitude of output electrical signal in dark and light conditions.

It could be seen that the self-oscillation appears once biased into the NDR region $(0.6 \mathrm{~V})$ and the frequency gradually increases with the bias voltage. It approaches the maximum when biased close to the third region. The oscillation frequency is slightly lower when the RTD-PD is illuminated. The output voltage is much higher compared to the non-oscillation state. The output voltage is $1.538 \mathrm{~V}$ as biased at $0.601 \mathrm{~V}$, which is about 250 times larger than the $6.15 \mathrm{mV}$ eye diagrams when biased at $0.59 \mathrm{~V}$. There is no obvious correlation between the current and the output amplitude when comparing with the I-V characteristic.

\section{2) RTD-PD oscillators}

In this part, we tested two oscillators with different oscillation frequencies. Firstly, a low frequency oscillator was utilized for the measurement. The peak voltage is around 0.72 $\mathrm{V}$ in the dark and the oscillation frequency is $5.47 \mathrm{GHz}$. A PRBS signal at a data rate of $50 \mathrm{Mbit} / \mathrm{s}$ was used to modulate the optical carrier. Output electrical eye diagrams could be obtained on the digital communication analyzer (Agilent $86100 \mathrm{C}$ ). In the whole PDR region (from $0 \mathrm{~V}$ to $0.71 \mathrm{~V}$ ), output signal indicated clear eye diagrams which are shown in Table 4 below.

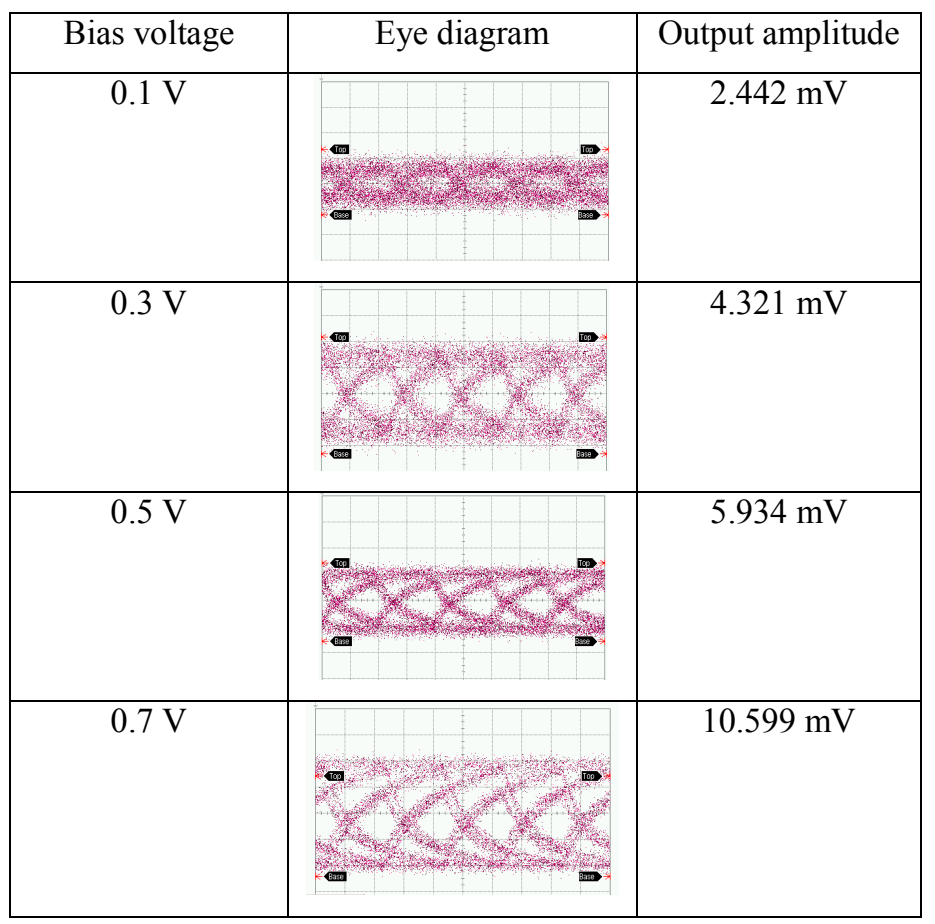

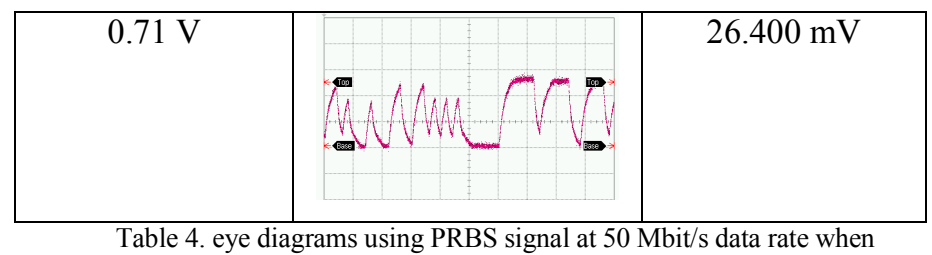

biased in the PDR region.

Like the results shown in Table 1, which represents the output eyes measured on the individual RTD-PD device, the amplitude of eyes increases with the bias voltage and the RTDPD oscillator shows similar features as a normal photodiode. The pattern length is corresponding to the optical injection data rates. The last pattern in the table shows the pattern lock mode of the eye diagram which indicates the output pattern of PRBS signal with random ' 1 ' and ' 0 ' levels.

Under the impact of light, the NDR region was shifted to the right. Once biased above $0.82 \mathrm{~V}$, the oscillator began to oscillate. Oscillations could be seen on top of the data pattern (such like biasing at $0.84 \mathrm{~V}$ ). It is important to choose a proper bias point because the ' 1 ' levels of the data pattern will drive the oscillator into oscillation mode when adequately biased. i.e. a ' 1 ' results in oscillation and a ' 0 ' does not. It could be noted that there are no oscillations on top of the pulses with lower amplitudes when biased at $0.84 \mathrm{~V}$ because of the insufficient bias. Too high a bias voltage level makes the oscillator work in the full oscillation state which makes the output signal ambiguous (such like biasing at $0.89 \mathrm{~V}$ ).

\begin{tabular}{|c|c|c|c|}
\hline Bias voltage & & Output signal pattern \\
\hline $0.82 \mathrm{~V}$ & & & \\
\hline $0.84 \mathrm{~V}$ & & & \\
\hline $0.89 \mathrm{~V}$ & & & \\
\hline
\end{tabular}

A higher frequency oscillator around $35 \mathrm{GHz}$ was then utilized to repeat the optical modulation measurement. Firstly, the I-V characteristic in both dark and light conditions was measured. The light source utilized a $\mathrm{CW}$ optical carrier at wavelength of $1310 \mathrm{~nm}$. 


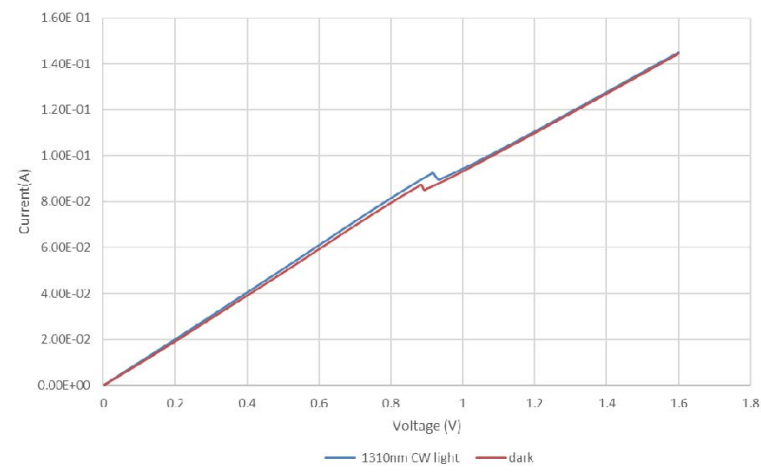

Fig. 5. I-V characteristic in dark and light conditions

The decreasing part in the middle of the I-V curve shown in Fig. 5 indicates the NDR regions. The NDR regions are not as obvious compared to the I-V characteristic of individual RTD devices, because of the external circuitry. As expected, it was found that the NDR region was shifted to the right in the presence of light and higher optical powers resulted in a larger shifting. In other words, higher bias voltage was required to achieve oscillation for higher optical powers because more light was absorbed in the emitter layer of the RTD device.

Data transmission using optical injection was then implemented. Once biasing the RTD-PD into the NDR region, a clear peak at around $35.1 \mathrm{GHz}$ would show on the spectrum analyzer. This is the carrier frequency generated by the RTDPD oscillator. The frequency could be tuned by changing the bias voltage. Increasing the bias voltage shifted the oscillation peak to higher frequencies. The tuning range was around tens of megahertz. The oscillation frequency moved to lower values in the presence of the light. The results are identical with the individual RTD-PD device, and also have been observed in [15]. The mechanism is that the accumulation of electron-hole pairs in the depletion region generated by the light decreases the bias across the RTD structure whose capacitance is increased accordingly [9], leading to the decrease of frequency.

Side bands located at both sides of the carrier appeared when the intensity modulated optical signal was injected into the device via the optical fiber. Fig. 6 presents the spectrum when the laser was modulated directly by a single subcarrier at a data rate of $200 \mathrm{Mbit} / \mathrm{s}$. The carrier frequency was fixed at $35.1 \mathrm{GHz}$. It was found that the distance between one side band to the carrier equaled half the data rate, due to the square wave nature of the incident signal used for amplitude modulation. As expected, increasing the modulation frequency would increase the frequency separation between two side bands and the amplitude of side bands decrease.

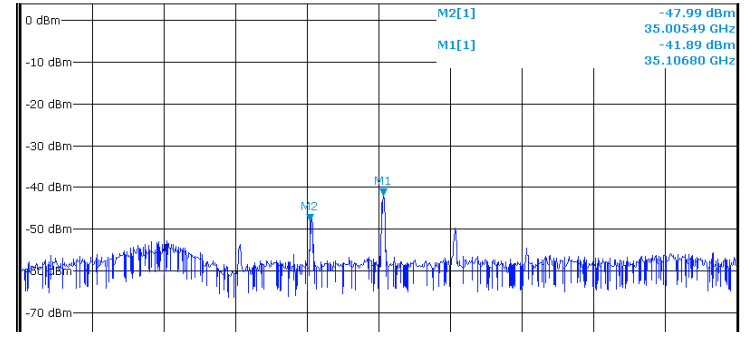

Fig. 6. Spectrum of intensity modulated RTD-PD oscillator using square wave signal at data rate $200 \mathrm{Mbit} / \mathrm{s}$
The sidebands employing the maximum data rate of 10 Gbit/s could be observed. When the data rate was increased further, the side bands began to get lost in the noise floor of the equipment. Despite still seeing these sidebands at high data rates, useful data transmission could only be achieved when the sideband amplitude is approximately $-3 \mathrm{~dB}$ lower than the carrier. Fig.7 shows the output time domain signal which indicates the data patterns mixed with the RTD-PD oscillations when injecting an optical signal, modulated by a PRBS signal at data rate of $80 \mathrm{Mbit} / \mathrm{s}$. The output electrical signal replicates the modulated optical signal with a carrier frequency at 35.1 GHz. The output signal has high output power due to the amplification effect of the RTD biased in oscillation mode. Oscillations clearly occur when the data pattern produces a ' 1 ' and remains outside the oscillation state for a ' 0 '. This is different from operation in the PDR region where more emphasis is on the input optical signal and the responsivity. When in the NDR region, the output is now limited by the RTD-PD power as opposed to the input optical signal.

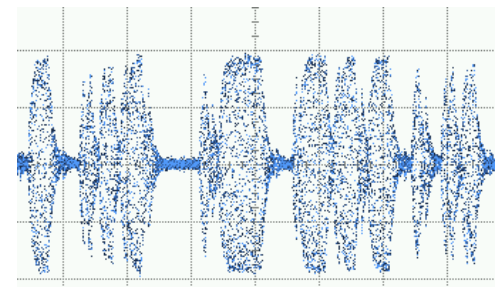

Fig.7. Output signal showing the data patterns at $80 \mathrm{Mbit} / \mathrm{s}$ mixed with oscillation.

Higher data rate transmission is expected in future work. It can be achieved, on the one hand, by improving the cutoff frequency, which is partly limited by the parasitic elements of the external circuit, and on the other hand, by increasing the output power, which can be achieved by improving the light coupling, modifying the thickness of the light absorption layer of the RTD-PD, and eliminating the attenuation effects coming from experimental set-up. Moreover, an oscillator employing double RTD-PDs was designed to increase the output power and the corresponding measurements have been primarily carried out [16].

\section{CONCLUSION}

This paper presents both an RTD based photodetector device and RTD-PD oscillators for the implementation of optical communications. Intensity modulated optical signals were applied to devices under test. Both the individual RTDPD device and oscillators have similar features to that of a photodiode when biased in the PDR region. Side bands induced by the input optical signal were observed on the spectrum analyzer and the corresponding time domain eye diagrams were obtained. Other optoelectronic characteristics have also been investigated, including that the NDC region could be shifted to higher bias voltages by light. Though the carrier frequency of the oscillators under test is still far from what is expected in the $\mathrm{THz}$ communication concept, work is ongoing to increase operating frequencies. It is promising to show that successful optical communications can be achieved using these RTD-PD based oscillators and higher data rate 
transmission can be reached by improving the cut-off frequency and output power of the devices, and experimental set-up in future work.

\section{ACKNOWLEDGMENT}

This work was performed as part of the iBROW project (www.ibrow-project.eu) which has received funding from the European Union's Horizon 2020 research and innovation programme under grant agreement No. 645369.

\section{REFERENCES}

[1] T. Kleine-Ostmann and T. Nagatsuma, "A review on terahertz communications research," Journal of Infrared, Millimeter, and Terahertz Waves, vol. 32, pp. 143-171, 2011.

[2] M. Takeru, K. Hidetoshi, S. Safumi, and A. Masahiro, "Oscillation up to $1.92 \mathrm{THz}$ in resonant tunneling diode by reduced conduction loss," Applied Physics Express, vol. 9, p. 024101, 2016.

[3] J. Figueiredo and B. Romeira, Resonant tunnelling optoelectronic circuits: Intech, 2010.

[4] T. Nagatsuma, "Terahertz communications technologies based on photonic and electronic approaches," in European Wireless, 2012. EW. 18th European Wireless Conference, 2012, pp. 1-4.

[5] H.-J. Song and T. Nagatsuma, "Present and future of terahertz communications," IEEE Transactions on Terahertz Science and Technology, vol. 1, pp. 256-263, 2011.

[6] J. Figueiredo, C. Stanley, A. Boyd, C. Ironside, S. McMeekin, and A. Leite, "Optical modulation in a resonant tunneling relaxation oscillator," Applied physics letters, vol. 74, pp. 1197-1199, 1999.

[7] J. Figueiredo, A. Boyd, C. Stanley, C. Ironside, S. McMeekin, and A. Leite, "Optical modulation at around $1550 \mathrm{~nm}$ in an InGaAlAs optical waveguide containing an InGaAs/AlAs resonant tunneling diode," Applied physics letters, vol. 75, pp. 3443-3445, 1999.

[8] A. Pfenning, F. Hartmann, F. Langer, S. Höfling, M. Kamp, and L. Worschech, "Cavity-enhanced resonant tunneling photodetector at telecommunication wavelengths," Applied Physics Letters, vol. 104, p. 101109, 2014.

[9] M. Kahn, J. Lasri, M. Orenstein, D. Ritter, and G. Eisenstein, "Phase-locking of an $\mathrm{InP} / \mathrm{InGaP} / \mathrm{InGaAs}$ resonant tunneling diode relaxation oscillator by direct optical injection," Solid-State Electronics, vol. 45, pp. 1827-1830, 2001.

[10] B. Romeira, J. Figueiredo, C. Ironside, A. Kelly, and T. Slight, "Optical injection locking of a resonant tunneling diode-optical waveguide photo-detector," Proc. ECIO 2010, 2010.

[11] T. Ramond, L. Hollberg, P. Juodawlkis, and S. Calawa, "Low-noise optical injection locking of a resonant tunneling diode to a stable optical frequency comb," Applied physics letters, vol. 90, p. 171124, 2007.

[12] T. Higgins, J. Harvey, D. Sturzebecher, A. Paolella, and R. Lux, "Direct optical frequency modulation and injection locking of resonant tunnel diode oscillator," Electronics Letters, vol. 28, pp. 1574-1576, 1992.

[13] J. Wang, L. Wang, C. Li, B. Romeira, and E. Wasige, "28 $\mathrm{GHz}$ MMIC resonant tunnelling diode oscillator of around
$1 \mathrm{~mW}$ output power," Electronics letters, vol. 49, pp. 816818, 2013.

[14] T. Moise, Y. C. Kao, L. Garrett, and J. Campbell, "Optically switched resonant tunneling diodes," Applied physics letters, vol. 66, pp. 1104-1106, 1995.

[15] J. Wang, "Monolithic microwave/millimetrewave integrated circuit resonant tunnelling diode sources with around a milliwatt output power," University of Glasgow, 2014.

[16] J. Tavares, L. Pessoa, J. Figueiredo, and H. Salgado, "Analysis of resonant tunnelling diode oscillators under optical modulation," in Transparent Optical Networks (ICTON), 2017 19th International Conference on, 2017, pp. $1-4$. 